\title{
Intussusception following enteroscopy and ileo-colonoscopy in an adult with acquired immune deficiency syndrome
}

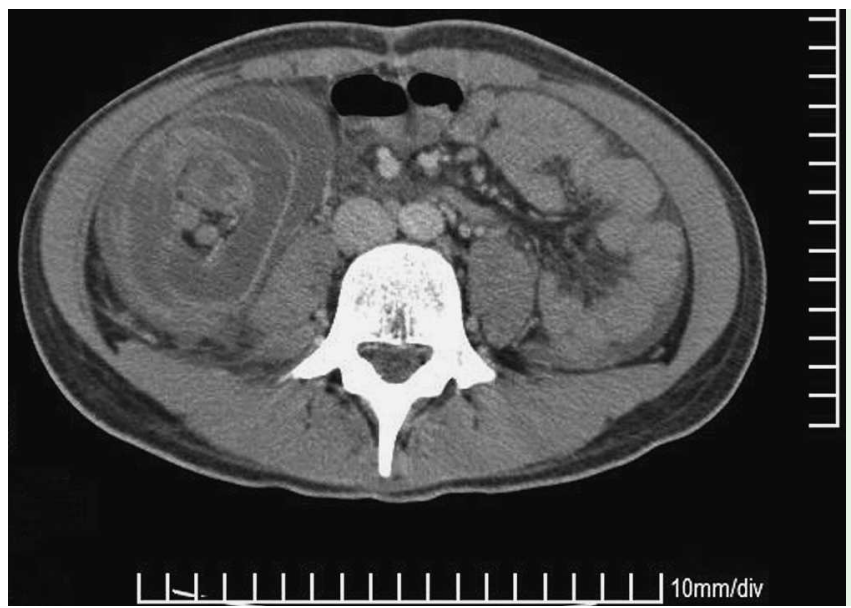

Fig. 1 Computed tomography showing the target sign in the right lumbar region.

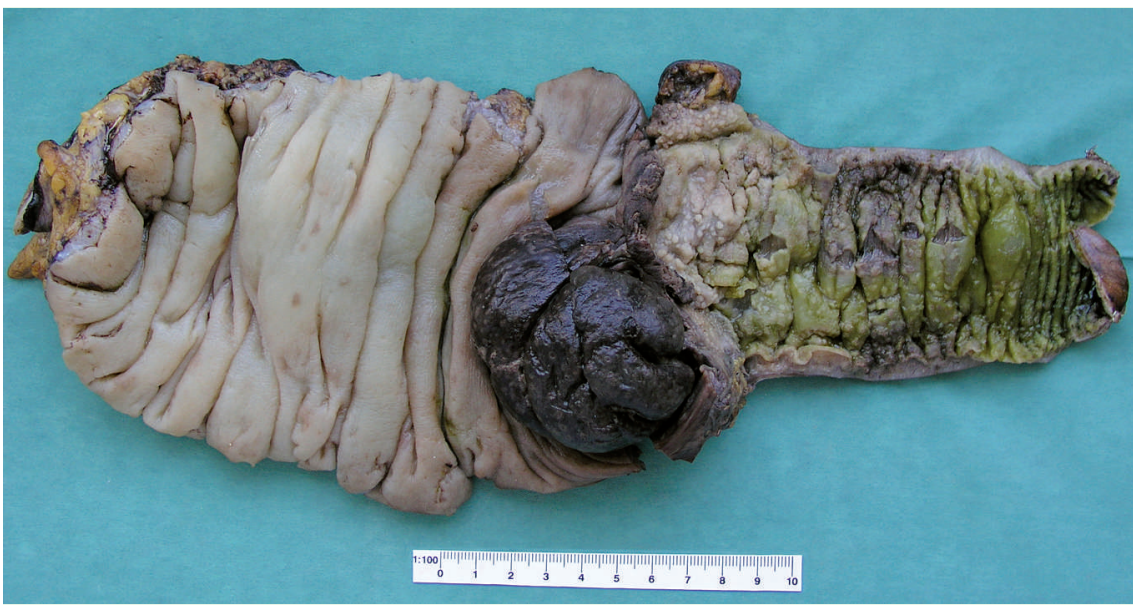

Fig. 3 Gross appearance of the resected region showing a well-defined ischemic lesion in the cecum and nodular hyperplasia at the terminal ileum.

A 19-year-old HIV infected man with advanced immunosuppression (CD4 counts of 140 cells $/ \mathrm{mL}$ ) was admitted to the department of internal medicine 4 months after primary infection, with diarrhea, epigastric pain, dysphagia, and weight loss. He was referred to us for an esophagogastroduodenoscopy which revealed Candida infection of the esophagus. Due to persistent epigastric pain, abdominal computed tomography (CT) was performed which revealed focal wall thickening of a jejunal loop. The patient under- went enteroscopy followed by ileocolonoscopy, both performed easily. Seven hours after the endoscopic procedures, the patient felt abdominal pain. On physical examination, he had localized tenderness in the right lumbar region. A plain abdominal radiograph did not show pneumoperitoneum. As the signs of peritoneal irritation persisted despite conservative management, the patient underwent an emergency abdominal CT which demonstrated a target mass in the right lumbar region ( $\bullet$ Fig. 1). A second, ur-

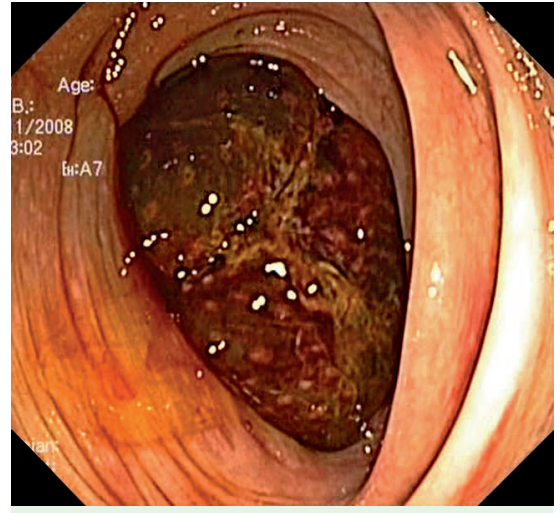

Fig. 2 Colonoscopy showing the mass-like lesion at the cecal base with bluish discoloration of the mucosa

gent colonoscopy showed a polypoid lesion with a diameter of $5 \mathrm{~cm}$ at the cecum with bluish discoloration of the mucosa ( $\bullet$ Fig. 2). On the basis of the CT images and the endoscopic appearance, a diagnosis of intussusception was made.

The patient underwent an emergency laparotomy and a right hemicolectomy was performed. Gross appearance of the resected specimen showed nodular hyperplasia at the terminal ileum and a well-defined ischemic lesion in the cecum ( Fig. 3). No tumor-like lesion to explain the intussusception was found either macroscopically or microscopically. The patient recovered uneventfully and was transferred to the internal medicine ward on the 5th postoperative day.

Intestinal intussusception is unusual in adults $[1,2]$. On searching the literature of the past 30 years, we found only one published case report of intussusception as a complication of colonoscopy [3]. Hyperperistalsis induced by the two sequential endoscopic procedures could be suggested as a cause of the intussusception in our case, with AIDS-associated gastrointestinal pathology being a risk factor.

Endoscopy_UCTN_Code_CPL_1AJ_2AB 
A. Theodoropoulou ${ }^{1}$, K. Konstantinidis ${ }^{1}$, S. Kteniadakis ${ }^{2}$, G. Tribonias ${ }^{1}$,

G. Tzanoudakis ${ }^{2}$, E. Vardas ${ }^{1}$,

K. Karmiris ${ }^{1}$, K. Psillakis ${ }^{3}$, E. Zois ${ }^{4}$,

E. Tzortzakakis ${ }^{5}$, G. A. Paspatis ${ }^{1}$

1 Department of Gastroentrology, Benizelion General Hospital, Heraklion, Crete, Greece

2 Department of Surgery, Benizelion General Hospital, Heraklion, Crete, Greece

3 Department of Computed Tomography, Benizelion General Hospital, Heraklion, Crete, Greece
4 Department of Pathology, Benizelion General Hospital, Heraklion, Crete,

Greece

5 Department of Internal Medicine, Benizelion General Hospital, Heraklion, Crete, Greece

\section{References}

1 Erkan $N$, Haciyanli $M$, Yildirim $M$ et al. Intussusception in adults: an unusual and challenging condition for surgeons. Int J Colorectal Dis 2005; 20: 452 - 456

2 Wang $L T, W u C C, Y u J C$ et al. Clinical entity and treatment strategies for adult intussusceptions: 20 years' experience. Dis Colon Rectum 2007; 50: 1941 - 1949

3 Yamazaki T, Okamoto H, Suda T et al. Intussusception in an adult after colonoscopy. Gastrointest Endosc 2000; 51: 356- 357
Bibliography

DOI 10.1055/s-0029-1214599

Endoscopy 2009; 41: E162-E163

(c) Georg Thieme Verlag KG Stuttgart · New York . ISSN 0013-726X

\section{Corresponding author}

\section{G. A. Paspatis, MD}

Benizelion General Hopsita

Department of Gastroenterology

L. Knossou

Heraklion

Crete

Greece 71409

Fax: +30-2810-368017

paspati@admin.teiher.gr 